\title{
Multilocus VNTR analysis of Mycobacterium ulcerans strains isolated in Côte d'Ivoire
}

\author{
Grossmann Marie-David Coulibaly-N'Golo ${ }^{1}$, Euloge Ekaza ${ }^{1}$, Bakary Coulibaly ${ }^{1}, N^{\prime}$ guetta Aka', \\ Raymond Kouassi N'Guessan ${ }^{1}$, Jean Michel Thiberge ${ }^{2}$, Valérie Caro ${ }^{2}$, Sylvain Brisse ${ }^{2}$, Mireille \\ Bretin-Dosso ${ }^{1}$
}

\author{
${ }^{1}$ Unité de microbiologie moléculaire, Département de Bactériologie- virologie, Institut Pasteur de Cote d'Ivoire, \\ Abidjan, Côte D'ivoire \\ ${ }^{2}$ Institut Pasteur, Genotyping of Pathogens and Public Health, Paris, France
}

\begin{abstract}
Introduction: Buruli ulcer, caused by Mycobacterium ulcerans, is endemic in more than 30 countries worldwide, with Côte d'Ivoire being among the most affected countries.

Methodology: We used seven variable number of tandem repeats (VNTR) markers and analyzed 114 samples from 11 Ivorian localities consisting of 33 bacterial strains and 81 clinical samples. Complete data sets at loci 1, 6, 9 and 33 were obtained for 18 of these strains (n = $15)$ and samples $(n=3)$ collected in each of the localities.

Results: All the strains had allelic profile [3113], corresponding to the previously described Atlantic Africa genotype.

Conclusion: Sequencing of PCR products at all loci showed no variation in sequence or repeat number, underlining the genetic monomorphism of M. ulcerans in Côte d'Ivoire.
\end{abstract}

Key words: Buruli ulcer; Mycobacterium ulcerans; Côte d'Ivoire; MIRU VNTR typing; sequencing

J Infect Dev Ctries 2011; 5(1):059-063.

(Received 05 July 2010 - Accepted 04 October 2010)

Copyright $\odot 2011$ Coulibaly et al. This is an open-access article distributed under the Creative Commons Attribution License, which permits unrestricted use, distribution, and reproduction in any medium, provided the original work is properly cited.

\section{Introduction}

Mycobacterium ulcerans infection causing Buruli ulcer (BU) is one of the most debilitating skin diseases in West Africa because of the unaesthetic sequelae and disabilities that it causes. BU is globally distributed, but its prevalence in West Africa is highest, and the disease is considered as re-emergent [1]. In western Africa, Côte d'Ivoire is the country that is the most severely affected, with more than 2,000 cases reported each year. BU is the second most prevalent mycobacterial disease of public health importance in Côte d'Ivoire, after tuberculosis.

$M$. ulcerans is an emerging environmental bacterium, and the epidemiology of $\mathrm{BU}$ is poorly understood. The precise mode of transmission and the environmental reservoirs and vectors of $M$. ulcerans remain unknown [2]. Recent studies showed that BU may be related to aquatic ecosystems, where water bugs could play an important role in the transmission, either as the ecological niche or as the mechanical vector of the bacteria [3-6]. Outbreaks are often unpredictable [3], and cases are focally distributed; endemic and non-endemic communities may be separated by only a few kilometers [1]. The clinical aspects of the disease are various [3,7] and are dependent on the patient or the geographic area.

Limited knowledge about BU epidemiology is partly due to the lack of methods able to reveal differences among isolates. To reach a better understanding of $M$. ulcerans epidemiology, strain typing methods are required. $M$. ulcerans is indeed a highly clonal pathogen [3] with limited genetic diversity on the basis of genomic sequences, which are much conserved even among isolates of distinct geographical regions [8]. The availability of complete genome sequences of $M$. ulcerans [9] allowed the identification of minisatellite markers including MIRUs [10] and other novel tandem repeat loci [11]. The use of such markers enabled the discrimination of $M$. ulcerans strains $[8,12,13]$. Variable number of tandem repeats (VNTR) typing has previously been used to analyze strains and environmental samples in 
Figure 1. Map of Cote d'Ivoire showing the localities from which samples and strains originated.

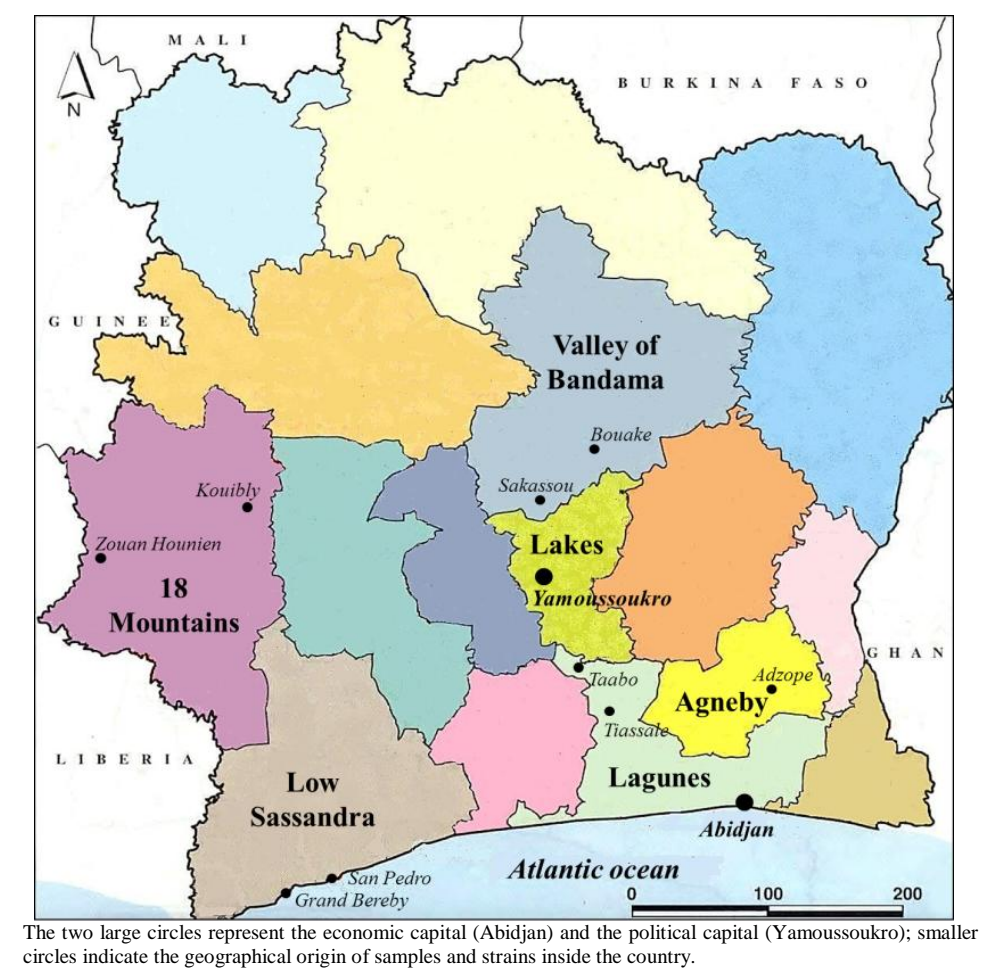

Ghana and Benin $[11,12]$. The aim of this study was to determine the diversity of $M$. ulcerans strains that circulate in Côte d'Ivoire, based on multilocus VNTR analysis.

\section{Methodology}

\section{Samples and bacteria}

A total of 114 samples from six major BU endemic regions in Côte d'Ivoire (from 11 different localities) and from different sources were collected between 2004 and 2008. Samples came either from the southern part of the country (regions of Lagunes, Agneby and Low-Sassandra), from the center (regions of Lakes and Valley of Bandama), or from the western part (region of 18 mountains; Figure 1).

The samples were made up of (i) 81 clinical samples including 62 exudates, 12 fine needles aspirates and 7 biopsies; (ii) 33 bacterial strains isolated from clinical samples at the Institut Pasteur, Côte d'Ivoire; and (iii) 7 reference DNA strains obtained from the Institute of Tropical Medicine, Antwerp, Belgium: ITM 9540 (Queensland, Australia), ITM 98-912 (China), ITM 5150 (Democratic Republic of Congo), ITM 97-0483 (Ghana), and 3 reference strains from Côte d'Ivoire (ITM 99-0006, ITM 94-0662 and ITM 94-0821).
DNA extraction and MIRU-VNTR typing

DNA from clinical samples was extracted as previously described $[14,15]$. DNA from bacterial strains was extracted by a physical method. Five colonies were harvested from Löwenstein-Jensen slants and suspended in a $1.5 \mathrm{ml}$ vial containing 200 $\mu \mathrm{l}$ of sterile water. Tubes were frozen at $-20^{\circ} \mathrm{C}$ for 20 minutes and subsequently boiled for 30 minutes in a heat block. The samples were then centrifuged for 10 minutes at $14,000 \mathrm{rpm}$ and the supernatant was collected and used as DNA source.

First the presence of M. ulcerans was confirmed in all samples by detecting the specific insertion sequences IS2404 and IS2606 by PCR as described previously [15-17]. Samples that were positive for the two targets IS2404 and IS2606 were analyzed by the MIRU-VNTR typing method. The following genetic markers were assayed: MIRU 1, MIRU 2, MIRU 5, VNTR 6, MIRU 9, MIRU 33 and ACC locus $[8,10-13,18]$. The minisatellites markers MIRU 1, VNTR 6, MIRU 9 and MIRU 33 were assayed for all samples of the study, whereas loci MIRU 2, MIRU 5 and ACC were targeted only for bacterial strains and 8 clinical samples that were positive for at least two of the previous loci. At least one of the control reference strains was used for each typing 
Table 1. Amplified samples obtained at the seven loci with the Ivoirian strains and samples.

\begin{tabular}{|c|c|c|c|}
\hline \multirow{2}{*}{ Locus } & \multicolumn{2}{|c|}{ Number of samples giving a positive PCR } & \multirow{2}{*}{ Total } \\
\cline { 2 - 3 } & Clinical samples & Bacterial strains & \\
\hline 1 & $07 / 81(8.6 \%)$ & $26 / 33(78.8 \%)$ & $33 / 114(28.9 \%)$ \\
\hline 6 & $09 / 81(11.1 \%)$ & $26 / 33(78.8 \%)$ & $35 / 114(30.7 \%)$ \\
\hline 9 & $39 / 81(48.1 \%)$ & $22 / 33(66.7 \%)$ & $61 / 114(53.5 \%)$ \\
\hline 33 & $15 / 81(18.5 \%)$ & $15 / 33(45.5 \%)$ & $30 / 114(26.3 \%)$ \\
\hline 2 & $3 / 8(37.5 \%)$ & $21 / 33(63.6 \%)$ & $24 / 41(58.5 \%)$ \\
\hline 5 & $4 / 8(50 \%)$ & $22 / 33(66.7 \%)$ & $26 / 41(63.4 \%)$ \\
\hline ACC & $3 / 8(37.5 \%)$ & $24 / 33(72.7 \%)$ & $27 / 41(65.9 \%)$ \\
\hline
\end{tabular}

reaction. PCR analysis was performed as described previously $[8,13]$.

\section{Sequencing}

All amplified products were sequenced using a 3730XL DNA Analyzer (Applied Biosystems, Courtaboeuf, France) after DNA quantification using a Nanodrop ND 8000 spectrophotometer (Thermo scientific, Wilmington, DE, USA). Sequence data analysis was performed using software BioNumerics v5 (Applied-Maths, Sint-Martens-Latem, Belgium), CLC Sequence Viewer 5.1.2 (CLC BIO) and Geneious Biomatters 4.5.4.

Permission for the study was granted by the National BU control program.

\section{Results and discussion}

Amplification of the four loci (1, 6, 9 and 33), which make it possible to discriminate $M$. ulcerans strains from various geographical origins $[8,10,11,18]$, revealed three profiles corresponding to the following genotypes: Atlantic Africa (profile [3113]), Southeast Asia (profile [1113]) and Asian (profile [1213]). The Ivorian samples (15 bacterial strains, three clinical samples) displayed the genotype "Atlantic Africa" that is widely distributed over Central and Western Africa [13]. Five reference strains (three from Côte d'Ivoire, one from Congo and one from Ghana) also matched the "Atlantic Africa" genotype. The reference strains ITM 9540 (Queensland, Australia) and ITM 98-912 from China had the genotypes "Southeast Asia" and "Asian", respectively. No new genotypic profile was found among our strains and samples. Each geographic region was represented in this complete data set. The genotypic profiles obtained with the reference controls were in agreement with previous findings [18]. Unfortunately, it was not possible to fully characterize $96(84 \%)$ of the samples, as no amplification was obtained for at least one of the four loci studied.

In addition, loci 2, 5 and ACC were assayed in 41 of the 114 samples analyzed (33 bacterial strains and 8 clinical samples), as well as the reference strains. The number of amplified samples for each target varied from 24 to 27 (Table I). Considering all seven loci, 236 PCR products were obtained, 211 of which could be sequenced and compared to reference strains (Table II). The low rate of PCR amplification of the MIRU/VNTR loci from DNA extracts prepared directly from clinical specimens could be expected, as the quantity of $M$. ulcerans DNA present in such extracts would be relatively low. However, the low rate of positive amplification obtained using DNA templates prepared from cultures in this study is unusual and may be related to sub-optimal laboratory conditions, rather than inherent limitations of the markers used. The number of analyzed sequences, the overall length of the repeated zone, and the number of tandem repeats by locus are given in Table II.

Overall, sequencing of PCR products from Ivorian isolates and clinical samples revealed no sequence difference at any locus compared to African reference strains.

At locus 1, twenty-eight sequences, including four African reference strains (ITM 5150, ITM 940821, ITM 97-0483, ITM 99-0006), were identical and showed three repeats as previously described $[10,18]$. The non-African reference strains ITM 9540 (Queensland, Australia) and ITM 98-912 (China) differed by having only one repeat and two single nucleotide polymorphisms (SNPs), respectively (Table II). At locus 6, the 25 Ivorian sequences, three African reference strains (ITM 97-0483, ITM 970006, ITM 5150) and an Australian reference strain (ITM 9540) had an identical 500 bp fragment, while the Chinese strain ITM 98-912 presented 5 SNPs and two repeats of a $40 \mathrm{nt}$ motif [11,18]. At locus 9, forty-four sequences, including that of the Ivorian 
Table 2. Sequence data analysis comparing Ivorian and reference strains used in this study

\begin{tabular}{|c|c|c|c|l|}
\hline Locus & $\begin{array}{c}\text { Sequenced } \\
\text { samples (No. of } \\
\text { reference strains } \\
\text { included) }\end{array}$ & $\begin{array}{c}\text { Number of } \\
\text { identical } \\
\text { sequences }\end{array}$ & $\begin{array}{c}\text { No. of repeats } \\
\text { [expected size] }\end{array}$ & \multicolumn{1}{|c|}{ Differences found* } \\
\hline $\mathbf{1}$ & $33(6)$ & 31 & 3 rep. [486 bp] & $\begin{array}{l}\text { ITM 98-9540: 1 rep. } \\
\text { ITM 98-912: 1 rep. + 2 SNPs }\end{array}$ \\
\hline $\mathbf{6}$ & $30(5)$ & 29 & 1 rep. [500 bp] & ITM 98-912: 2 rep. + 5 SNPs \\
\hline $\mathbf{9}$ & $45(2)$ & 44 & 1 rep. [440 bp] & ITM 98-912: 4 SNPs \\
\hline $\mathbf{3 3}$ & $25(2)$ & 24 & 3 rep. [850 bp] & ITM 98-912: 6 SNPs \\
\hline $\mathbf{2}$ & $24(0)$ & 24 & 2 rep. [145 bp] & None. \\
\hline $\mathbf{5}$ & $28(5)$ & 26 & 1 rep. [625 bp] & $\begin{array}{l}\text { ITM 98-9540: 1 SNP } \\
\text { ITM 98-912: 2 SNPs }\end{array}$ \\
\hline ACC & $26(4)$ & 25 & 34 rep. [375 bp] & ITM 9540: 1 SNP \\
\hline
\end{tabular}

strain ITM 94-0821, were identical with a single repeat unit in the PCR product of about $440 \mathrm{bp}$ [13]. The reference strain ITM 98-912 (China) differed by four SNPs at this locus. Finally, at locus 33, twentyfive sequences of the 850-bp PCR product, including that of ITM-94-0821, were identical, with a 41-nt sequence repeated three times as described [13]. The Chinese reference strain ITM 98-912 differed by six SNPs.

The additional loci were also investigated by sequencing. At locus 2, all sequences were identical, with no tandem repeat sequences as described [10], even though two "ATCGAT" motifs (thus smaller than the entire MIRU repeat unit) were observed. At locus 5, twenty-six of our sequences showed 100\% identity, while the two non-African reference strains showed 1 and 2 SNPs, respectively. No repeated sequence was found for this locus consistent with previous reports [10]. Finally, at the ACC locus, the sequence of the expected $375 \mathrm{bp}$ fragment was identical in 25 PCR products, with 34 copies of the heterogeneous trinucleotide array, consistent with a previous study [8]. The Australian reference strain ITM 9540 presented one SNP at this locus.

In conclusion, no SNP or repeat number difference was detected in the investigated loci. The high level of sequence conservation found at the 7 loci mentioned in this study shows that $M$. ulcerans populations from Côte d'Ivoire harbor extremely low levels of polymorphism, similar to the situation in other African countries [19-21].

This is the first extensive study on the genetic diversity of $M$. ulcerans in the Côte d'Ivoire. Our results demonstrate that the Ivorian strains correspond to a genotype previously described in other African countries. M. ulcerans exhibits a high level of sequence conservation, especially among isolates within a given geographic origin. As even the use of minisatellite markers showed a limited ability to discriminate among $M$. ulcerans strains, more discriminatory marker sets such as SNP typing assays with a high-resolution [22,23] will be needed to decipher the molecular epidemiology of $M$. ulcerans strains in Côte d'Ivoire and other endemic regions.

\section{Acknowledgements}

We thank Françoise Portaels and Pieter Stragier from the Institute of Tropical Medicine, Belgium, for providing reference strains DNA (ITM 9540, ITM 98-912, ITM 5150, ITM 97-0483, ITM 99-0006, ITM 94-0662, ITM 94-0821). Two anonymous reviewers contributed to the improvement of the manuscript. We also thank the "Division International" of Institut Pasteur for their administrative assistance.

This work was supported financially by the Institut Pasteur de Côte d'Ivoire, Institut Pasteur (Paris, France) and the Institut de Veille Sanitaire (Saint-Maurice, France).

\section{References}

1. WHO (2008) Buruli ulcer: progress report, 2004-2008. WHO Weekly epidemiological record 83: 145-156. Available:

http://www.who.int/entity/wer/2008/wer8317/en/index.html. Accessed 25 August 2008.

2. Lavender C J, Stinear TP, Johnson PDR, Azuolas J, Benbow ME, Wallace JR, Fyfe-Janet AM (2008) Evaluation of VNTR typing for the identification of Mycobacterium ulcerans in environmental samples from Victoria, Australia. FEMS Microbiol Lett 287: 250-255.

3. Demangel C, Stinear TP, Cole ST (2009) Buruli ulcer: reductive evolution enhances pathogenicity of Mycobacterium ulcerans. Nat Rev Microbiol 10: 50-60.

4. Marsollier L, André J-PS, Frigui W, Reysset G, Milon G, Carbonnelle B, Aubry J, Cole ST (2007) Early trafficking events of Mycobacterium ulcerans within Naucoris cimicoides. Cell Microbiol 9: 347-355. 
5. Silva MT, Portaels F, Pedrosa J (2007) Aquatic Insects and Mycobacterium ulcerans: An Association Relevant to Buruli ulcer Control? Plos Medicine 63: 229-231.

6. Mosi L, Williamson H, Wallace JR, Merritt RW, Small PLC (2008) Persistent association of Mycobacterium ulcerans with west African predaceous insects of the family Belostomatidae. Appl Environmental Microbiol 74: 70367042.

7. Portaels F, Silva MT, Meyers WM (2009) Buruli ulcer. Clin Dermatol 27: 291-305.

8. Ablordey A, Fonteyne P-A, Stragier P, Vandamme P, Portaels F (2007) Identification of a new variable number tandem repeat locus in Mycobacterium ulcerans for potential strain discrimination among African isolates. Clin Microbiol Infect 13: 734-736.

9. Stinear TP, Seemann T, Pidot S, Frigui W, Reysset G, Garnier T, Meurice G, Simon D, Bouchier C, Laurence M, Tichit M, Porter JL, Ryan J, Johnson PDR, Davies JK, Jenkin GA, Small PLC, Jones LM, Tekaia F, Laval F, Daffé M, Parkhill J, Cole ST (2007) Reductive evolution and niche adaptation inferred from the genome of Mycobacterium ulcerans, the causative agent of Buruli ulcer. Gen Res 17: 192-200.

10. Stragier P, Ablordey A, Meyers WM, Portaels F (2005) Genotyping Mycobacterium ulcerans and Mycobacterium marinum by using mycobacterial interspersed repetitive units. J Bacteriol 187: 1639-1647.

11. Ablordey A, Swings J, Hubans C, Chemlal K, Locht C, Portaels F, Supply P (2005) Multilocus variable-number tandem repeat typing of Mycobacterium ulcerans. J Clin Microbiol 43: 1546-1551.

12. Hilty M, Yeboah-Manu D, Boakye D, Mensah-Quainoo E, Rondini S, Schelling E, Ofori-Adjei D, Portaels F, Zinsstag J, Pluschke G (2006) Genetic diversity in Mycobacterium ulcerans isolates from Ghana revealed by a newly identified locus containing a variable number of tandem repeats. $\mathrm{J}$ Bacteriol 188: 1462-1465.

13. Stragier P, Ablordey A, Bayonne LM, Lugor YL, Sindani IS, Suykerbuyk P, Wabinga H, Meyers WM, Portaels F (2006) Heterogeneity among Mycobacterium ulcerans isolates from Africa. Emerg Infect Dis 12: 844-847.

14. Ekaza E, Kacou-N'douba A, Oniangue NC, Ehuie P, N'Guessan KR, Aka N, Bouzid SA, Faye-Kette H, Dosso M (2004) Contribution of genic amplification in the detection of M. ulcerans in exudates and in cutaneous biopsies in Cote d'Ivoire. Bull Soc Path Exo 97: 95-96.

15. Coulibaly B, Coulibaly-N'Golo M-DG, Ekaza E, Aka N, N'Guessan KR, Baudryard A, Assandé J-M, Trébissou N, Guédé-Guina F, Dosso M (2010) Implementation of in vitro culture of Mycobacterium ulcerans from clinical samples versus detection of acid fast bacilli and bacterial genome in Abidjan, Cote d'Ivoire. Bull Soc Path Exo 103: 2-7.

16. Ross BC, Johnson PD, Oppedisano F, Marino L, Sievers A, Stinear T, Hayman JA, Veitch MG, Robins-Browne RM (1997) Mycobacterium ulcerans in environmental samples during an outbreak of ulcerative diseases. Appl Environmental Microbiol 63: 4135-4138.

17. Stinear TP, Ross B, Davis J, Marino L, Robins-Browne R, Oppedinaso F, Sievers A, Johnson PD (1999) Identification and characterization of IS2404 and IS2606: two distinct repeated sequences for detection of Mycobacterium ulcerans by PCR. J Clin Microbiol 37: 1018-1023.

18. Stragier P, Ablordey A., Durnez L, Portaels F (2007) VNTR analysis differentiates Mycobacterium ulcerans and IS2404 positive mycobacteria. Syst Appl Microbiol 30: 525-530.

19. Stinear TP, Jenkin GA, Johnson PD, Davies JK (2000) Comparative genetic analysis of Mycobacterium ulcerans and Mycobacterium marinum reveals evidence of recent divergence. J Bacteriol 182: 6322-6330.

20. Stinear TP, Hong H, Frigui W, Pryor MJ, Brosch R, Garnier T, Leadlay PF, Cole ST (2005) Common evolutionary origin for the unstable virulence plasmid pMUM found in geographically diverse strains of Mycobacterium ulcerans. J Bacteriol 187: 1668-1676.

21. Yip MJ, Porter JL, Fyfe-Janet AM, Lavender CJ, Portaels F, Rhodes M, Kator H, Colorni A, Jenkin GA, Stinear T (2007) Evolution of Mycobacterium ulcerans and other mycolactone-producing mycobacteria from a common Mycobacterium marinum progenitor. J Bacteriol 189: 20212029.

22. Qi W, Käser M, Röltgen K, Yeboah-Manu D, Pluschke G (2009) Genomic diversity and evolution of Mycobacterium ulcerans revealed by next-generation sequencing. PLoS Pathog, 5 (9): e1000580. Published online 2009 September 11. doi:10.1371/journal.ppat.1000580

23. Käser M, Hauser J, Pluschke G (2009) Single nucleotide polymorphisms on the road to strain differentiation in Mycobacterium ulcerans. J Clin Microbiol 47: 3647-3652.

\section{Corresponding author}

Euloge Ekaza

Institut Pasteur de Côte d'Ivoire

Côte d'Ivoire

Email: ekazae@yahoo.fr

Conflict of interests: No conflict of interests is declared. 